\title{
Parenthood and depression: is childlessness similar to sonlessness among Chinese seniors?
}

\author{
MAJA DJUNDEVA*, TOM EMERY*† and PEARL A. DYKSTRA*
}

\begin{abstract}
We investigate how the mental health of older adults $(60-85)$ is associated with childlessness and sonlessness in China, where gender-biased filial expectations and strong son preference exist. The China Family Panel Study (2012, N=6,021) and ordinary least squares regression models are used to investigate the relationship between depression (Center for Epidemiologic Studies - Depression scale) and parental status, distinguishing between childless, parents of both sons and daughters, parents of only sons and parents of only daughters. Arguing that modernisation shapes gender preferences for children as well as formal care and pension provisions for older adults, we find a sharp rural-urban divide in the relationship between parental status and depression. Just having a son is not what matters as the best faring groups are parents who have both sons and daughters, regardless of the number of children. Rural childless and sonless are similar, whereas in urban areas parental status is not so salient, supporting modernisation theory.
\end{abstract}

KEY WORDS - China, sonlessness, intergenerational relations, depression, mental health, parenthood.

\section{Introduction}

In 2015 , people over 65 made up approximately 1 o per cent of the Chinese population, constituting about 137 million people (Central Intelligence Agency 2015). The proportion of the old has been steadily growing over time from 7.9 per cent of the total population in 2007 and is projected to grow to over 200 million in 2025 , or nearly one-third of the population (United Nations 2007, 2008). Concerns arise for the psychological wellbeing of older adults in China as studies report prevalence of depression

* Department of Public Administration and Sociology, Erasmus University, Rotterdam, The Netherlands.

$\dagger$ Netherlands Interdisciplinary Demographic Institute, The Hague, The Netherlands. 
in older people, ranging from 3.86 to 30.8 per cent (Chen et al. 1999; Gao et al. 2009; Lai et al. 2010 ; Wen et al. 2010). It is well established that depression is correlated with worse physical health and with higher mortality (see reviews by Pan et al. 2011; Wulsin, Vaillant and Wells 1999). Depression in older Chinese adults has been associated with diminished quality of life (Feng, Guo and Liu 2016), and studies found that it is more prevalent in women, those living in rural areas and among the least educated older adults (Liu, Meng and Tang 2004; Wang et al. 2006). Social stigma of mental illness in China heavily influences help-seeking behaviours of older adults and the reported prevalence rates, and emotional distress is frequently, if at all, expressed 'in an idiom of bodily complaints' (Lim et al. 2011 ). An under-developed social welfare system and a Confucian tradition put great emphasis on children taking care of older parents, thus fostering strong links between the presence of adult children and the mental health of older adults (Chen and Silverstein 2000; Cong and Silverstein 2008; Guo, Chi and Silverstein 2016).

Parenthood 'emerges as a key organiser of the life course and a major factor in social integration' (Dykstra and Hagestad 2007: 1275), and in China the presence of children is essential to continued survival when health declines in old age. Adult children next to grandchildren are central in the support systems for older adults in China, as they an important source of emotional, instrumental and financial support (Cong and Silverstein 2011a, 2012; Lee and Xiao 1998). Co-residence of older adults with usually married children (Chu, Xie and $\mathrm{Yu} 2011$; Whyte 2005) and upward monetary transfers from adult children to parents are frequent and expected, especially in rural areas (Cong and Silverstein 2011 b; Guo, Chi and Silverstein 2012; Xie and Zhu 2009). Moreover, children in China are an 'old-age insurance' because in many regions a lack of pensions, health care and social services makes older adults much more dependent on adult offspring compared to many Western welfare states. In addition, the Chinese pronatalist culture puts great emphasis on the milestone of becoming a parent, as part of 'normal expectable life'. Therefore, having no children that would offer support might have substantial psychological consequences for older adults.

Previous studies of depression in older Chinese have focused on the differences between parents and non-parents, omitting the importance of the gender composition of children [with the exception of studies investigating physical health (Zeng et al. 2016) and life satisfaction (Zhang and Liu 2007)]. Traditional filial expectations in China are gender-biased and filial piety is particularly expected from sons and their spouses (Cong and Silverstein 2012). Thus, not only the absence of children, but specifically the absence of sons might pose a serious disadvantage for parental health, 
especially in a culture where filial expectations are in line with a patriarchal family tradition that promotes patrilocal residence and patrilineal transfer of assets such as housing and land (Whyte 2004). The strong preference for sons, the extent to which parents have gender-specific differential treatment of children, as well as the desired number of children (as older adults had children before the 'one-child policy') vary across China given that urban-rural differences in culture, social policy and economic development have increased over time (Whyte 2004, 2010).

Modernisation shapes gender preferences for children, and China offers a unique context to investigate how the intersection of cultural norms and social institutions impact the mental health of its older population. The level of modernisation differs across China, with urban areas being much more developed compared to rural, thus presenting an opportunity to find out how children might have different psychological benefits for older adults in areas that vary in their degree of modernisation. We set out to investigate what implications modernisation and individualisation have for the mental health of older adults, focusing on parenthood as a central feature of family life in China. In this study we are interested in establishing the ways in which the urban-rural divide in China shapes the experience and consequences of parenthood (being a parent of sons or daughters) or not having any children (childless) for the mental health of older adults. How do the childless fare in comparison with parents who do not have sons? Are the childless worse off than all parents, or are they similar to parents that have only daughters with regards to mental health?

The rural-urban difference is the most fundamental socio-economic and demographic marker and a major driving force behind inequalities in China (Liu, Hsiao and Eggleston 1999; Wu and Treiman 2004). Therefore, the Chinese social context provides an opportunity to investigate how the processes of modernisation and individualisation can shape the relationship between mental health and parenthood in later life. The levels of modernisation and industrialisation in China have a large impact on family structure and intergenerational relationships, but to date, previous research has been limited to investigating the mental health of either childless urban residents or older parents living in rural areas. For example, surveys were administered only to older urban childless residents (Cheng et al. 2014; Zhang and Liu 2007). Moreover, studies compared the older childless with parents, neglecting the potential differential effect of sons and daughters (Chou and Chi 2004). Note, however, that the implications of older parents' mental health have been well-documented using large-scale surveys, but focusing on the rural population (Chen and Silverstein 2000; Cong and Silverstein 2008; Guo 2014; Guo, Aranda and Silverstein 2009; Guo, Chi and Silverstein 2012, 2016). 


\section{Background}

\section{Childlessness in China}

Until the 1960s, the Chinese government encouraged families to have as many children as possible as demographic expansion was considered key to bringing greater economic prosperity. In the 1970 , citizens were encouraged to have only two children, and between $195^{2}$ and 1979 (the 'one child policy' was introduced in 1979), the Chinese total fertility rate fell from 6.5 to 2.75 (Potts 2006). Chinese tradition puts a big emphasis on the continuation of family lines (Chu 1991), but the proportion of childless adults is increasing, and very little is known about the potential disadvantages of childlessness in later life in China. In the future, due to the marriage squeeze on males and the gender imbalance resulting from son preference, discrimination against girls, sex-selective abortion and strict fertility policies (Poston and Glover 2005), involuntary childlessness is set to rise.

The proportion of childlessness among older individuals in China is estimated to be approximately 4.6 per cent (Zhang and Liu 2007). In urban areas it is thought to be much higher, with 10 per cent childlessness estimated in Hong Kong of older adults in 2003 (Chou and Chi 2004). Yet this is expected to grow and predictions based on the 2005 Census from the $40-44$ age group estimate that 25.7 per cent of women in cities will remain childless when they reach the age of $5^{\circ}$ (Lui 2010). Childlessness is an outcome of many events and processes over a person's lifecourse. Pathways leading into childlessness in China are not particularly distinctive from elsewhere, despite marriage being almost universal for older cohorts (Dykstra and Hagestad 2007; Yi, Vaupel and Yashin 1985; Zhang and Liu 2007). Firstly, unmarried adults are not likely to have biological children, thus children born out of wedlock in the past were considered illegitimate, and in most cases were placed in orphanages (Johnson, Banghan and Liyao 1998). The unmarried also rarely adopted children and frequently remained childless in old age. Secondly, some married individuals remained childless involuntarily due to infertility issues, and thirdly, a small proportion of married parents have outlived their adult children. The presentday older adults experienced the events of the Second World War, the Great Famine (1959-1961) and the Cultural Revolution (1966-1976), and children born and raised in those periods had a higher risk of not reaching adulthood (Hesketh and Wei 1997; Song 2009).

One reason why childlessness might be associated with depression is due to the stigma of childlessness in the pronatalist Chinese culture (Johnson 2004; Zhang and Liu 2007). This is especially pertinent for married women as the patriarchal culture puts emphasis on the duty of wives to continue the family line. Research shows that the childless over 6o in Hong Kong are lonelier 
(both women and men) and more depressed (only men) compared to parents (Chou and Chi 2004). Although the childless are more likely to be never married and less likely to be currently married than the parents of adult children, the findings from the study of Chou and Chi (2004) revealed no differences in loneliness between the married and unmarried childless. Another study of Zhang and Liu (2007) found that the childless elderly were less satisfied with their lives and felt more anxious and lonely compared to parents, but they did not necessarily feel significantly more useless.

Another reason why childlessness might lead to low levels of mental wellbeing and higher depression is because of a lack of social support, as alternative sources of support such as health and community resources are limited, especially in rural areas in China (Shen and Williamson 2010; Yip and Hsiao 2009). Despite having formal rights as welfare recipients eligible for nursing home care in urban areas, older childless adults in China are frequently stigmatised, and sometimes discriminated against. It is not uncommon for older childless adults to have limited access to formal care homes and services due to legal and institutional barriers that require a child to serve as a guarantor (Feng et al. 2012). Older individuals can expect support from extended kin when they live in the same household (Cooney and Di 1999). The limited research of the childless in China provides no information on support from other kin to the childless. Presumably, having children to take care of parents in old age by providing social support in the form of personal care and financial support, as well as emotional support, is of essence to the wellbeing of older individuals (Chen and Silverstein 2000; Djundeva et al. 2015; Silverstein, Chen and Heller 1996; Tiikkainen and Heikkinen 2005). Childlessness might lead to low levels of mental wellbeing and more depressive symptoms because of a lack of social support. Taking into consideration the possible lack of social support and stigma of the childless status, we expect that the childless will be more depressed than parents (Hypothesis 1).

\section{Son preferences and mental health}

Sex-testing technologies in China became widespread in the early 1980s, but a large volume of research has established that parental son preference existed long before sex-selection became available (e.g. Arnold and Zhaoxiang 1986; Chen, Xie and Liu 2007; Coale 1991; Poston 2002). Previous studies on preferences for the gender of children in developing countries showed that sons are mainly wanted for (a) continuity of the family line and (b) financial and practical help; whereas daughters are desired because (a) they help to care for younger siblings, (b) they engage in household tasks that reduce the workload of the parents, (c) 
they engage in paid labour and contribute economically to the household, and (d) they provide care for their parents in old age that is more reliable and of higher quality than is available from sons (Arnold and Kuo 1984; Cleland, Verrall and Vaessen 1983).

Parents' gender preferences for children are embedded in cultural and religious traditions and community norms, and children of a particular sex are desired in order to provide a social security system where insurance programmes and provisions for illness and old age are lacking. Even in China, many families consider it important to have at least one daughter among their children (Johnson, Banghan and Liyao 1998). The desire for a mixed-gender offspring has been ascribed as a strategy for 'uncertainty reduction' in old age (Friedman, Hechter and Kanazawa 1994), as sons and daughters are expected to provide different types of support. With regards to mental health, a study from rural China reports that receiving and providing monetary support to adult children is related to lower levels of depression for mothers and fathers (Guo, Chi and Silverstein 2016). Similarly, depressive symptoms are reduced over time by receiving instrumental support (household chores and personal care) from daughters-in-law and increase when such support comes from sons (Cong and Silverstein 2008).

Research done on survey data from urban China has established that sons are more likely to provide financial support to their older parents, whereas daughters are more likely to provide gifts and assistance in daily living to their older parents, but even more to in-laws (Cooney and Di 1999; Sun 2002; Zhan 2004). Next to the strong son preference among older adults, it is likely that parents who did not succeed in having a son are disappointed, and lack both adequate financial and emotional support, which in turn is negatively associated with mental health. Being sonless might have similar associations with mental health as being childless, whereas parents of sons are likely to have daughters-in-law. They can further offer parents-in-law instrumental and emotional support, therefore having an additional positive influence on parental health. As daughters are supposed to marry off and contribute to the husband's family in the role of daughters-in-law (Bian, Logan and Bian 1998), parents without sons who only have daughters are more likely to be similar to childless adults than to those who have only sons, or a combination of sons and daughters. We expect that parents who have only daughters will be more depressed than those who had only sons or both daughters and sons (Hypothesis 2).

\section{Parenthood and modernisation}

The vast regional economic differences between coastal and inland regions in China have been a product of the different pace of modernisation and 
urbanisation over the past few decades. Thus, the patrilocal, patrilineal and patriarchal aspects of Chinese culture have remained more salient for rural families compared to urban ones (Whyte 2010). Due to increased commodification of many basic social services that are provided by enterprises to their employees, older rural residents have limited access compared with urban residents to health care, including nursing care and care homes (Pei and Tang 2012). A strict system of household registration control (hukou) established before the 1980s has kept migration under tight regulation, excluding the majority of rural migrants from the urban social security system and basic health care, old-age pension, work injury insurance or educational rights for their children (Chan and Zhang 1999). All this has put a greater emphasis on having adult children for older adults who live in rural areas, as they are more likely to suffer being childless because of a lack of social support, as well as having children for rural migrants who are excluded from the urban hukou welfare system. In addition, a study has shown that rural residents are probably more likely to experience greater stigma when they remain childless due to cultural stereotypes (Zhan, Luo and Chen 2012). Therefore, we expect that the difference in depression will be greater between the rural childless (and the rural childless migrants) and the rural parents, compared to the difference between their urban counterparts (Hypothesis 3 ).

The norm of receiving support in old age primarily from sons may have been undermined by dramatic demographic, economic and cultural changes occurring over the last several decades in China, especially in urban areas. Xie and Zhu (2009) examined gender differences in adult children's financial support to parents in three large cities (Shanghai, Wuhan and Xi'an) and found that married daughters, especially those living with parents, provide more financial support to parents than married sons do. This significant gender difference can be primarily explained by daughters' resources, such as education and income, which have enabled them to assume increasingly active roles in the support networks of their elderly parents. Conversely, in rural areas, because of large-scale labour-force migration, it is common that adult sons have moved away from their ageing parents, reducing the availability of sons (Giles and $\mathrm{Mu} 2007$ ). Studies researching rural residents reported that although daughters are less available to provide financial support to their own aged parents than sons, they still provide instrumental and emotional support to biological parents (Zhang and Wang 2010).

In rural areas, in particular, when daughters married they were considered to be a part of their husband's household. Once married, girls were in most cases leaving the parental household, so parents frequently had gender-specific treatment when deciding about children's education (Luo 
2012). Therefore, despite major changes in contemporary China, there is clear evidence that older parents have provided differential treatment in resource allocation among children based on children's gender, usually by offering more educational and migration opportunities to male children (Chu and $\mathrm{Yu}$ 2009). Because much of the livelihood of the rural population depends on agricultural work, there is a markedly stronger preference for sons in rural areas compared to urban areas (Arnold and Zhaoxiang 1986). A shift has occurred in the 'gender system' that shapes prevalent gender roles and dictates which opportunities are available to men and women (e.g. in health care, education and the labour market). This shift made the relative importance of sons over daughters for parents diminish in urban areas as daughters of younger cohorts have started to benefit from a greater access to resources. Based on that, we expect that the older adults without sons (who have only daughters) will be more disadvantaged in rural areas or when they are rural migrants than urban residents, therefore making the difference in depression levels greater between the rural sonless parents compared to the urban (Hypothesis 4 ).

\section{Method}

\section{Sample}

To investigate the relationship between parental status and depression, and how it differs for different groups based on the place of residence and migration status of older adults, we used the second wave of the Chinese Family Panel Study (CFPS) collected in 2012. The CFPS is a national longitudinal household panel study conducted in 25 Chinese provinces (http://www. isss.edu.cn/cfps/EN/) covering the economic activities, educational attainment, family relationships, migration, and physical and mental health of the Chinese population. The target sample of CFPS consists of 16 ,ooo households in 25 provinces in China (excluding Hong Kong, Macao, Xinjiang, Tibet, Qinghai, Inner Mongolia, Ningxia and Hainan). The population of these 25 provinces covers 95 per cent of the Chinese total population, therefore CPFS is regarded as a nationally representative sample. Data were collected via face-to-face interviews (computer-assisted personal interviewing) with the sampled households' family members (CFPS User's Manual 2012) in a joint project implemented by the National Population and Family Planning Commission, the State Bureau of Statistics, Shanghai University, Sun Yat-Sen University and the University of Michigan.

The original sample of 26,338 respondents was restricted to respondents between the ages of 60 and 85 who did not have missing data on any of the measures of interest. Out of 6,282 respondents in the age range, 6,022 had 
complete information on parental status, depression and the rest of the variables, with data missing from 4.15 per cent of respondents. The oldest old $(>85)$ were excluded as the relationship between parental status and mental health is probably different in this group and depression is more likely to be related to decline in cognitive and physical health (Stek et al. 2004). The descriptive statistics and the coding scheme for all variables used in the analyses are shown in Table 1.

\section{Measures and analytic strategy}

Depression. The dependent variable was the severity of depressive symptoms and was measured with the 20-item Center for Epidemiologic Studies Depression (CES-D) scale (Radloff 1977). The items referred to sadness, loss of interest, appetite, sleep, concentration, worthlessness, fatigue and movement. Several studies have investigated the reliability and validity of the Chinese version of the CES-D scale and found that it shows good reliability and validity across ages in the urban population (Zhang and Li 2011; Zhang et al. 2010), and more specifically in a community elderly population (Feng, Guo and Liu 2016). After we reversed the coding of positive affect items, we coded the frequency with which the respondent had experienced each symptom in the past into a summary score according to the CES-D protocol (CESD-R Explanation 2008) as follows: o (almost never, less than one day), 1 (sometimes, one or two days), 2 (often, three or four days) and 3 (most of the time, five to seven days). The sum of the scored variables resulted in a depression score ranging from o to 6o, with a higher score indicating more depressive symptoms. The scale reliability coefficient for the 20 items was high $(\alpha=0.85)$.

Parental status. Respondents were asked information for up to ten children, including whether children were alive, and about the gender of each child as well as other characteristics. The respondents in the data are considered childless if they reported not having even one child alive in 2012. This means that individuals are considered childless if they have never been biological parents (9o.89 per cent of those who were childless) and those who have outlived their children (9.11 per cent of those who were childless). Because of the small number of parents who have outlived their children and because the results do not differ significantly when we exclude them, they have been included in the analyses. All of the respondents were classified into one of the four categories: (a) they had both sons and daughters, (b) only sons, (c) only daughters or (d) were childless. The number of children was used as a control variable and coded as dummy variables, 
T А В L E 1. Socio-demographic information of the respondents by parental status

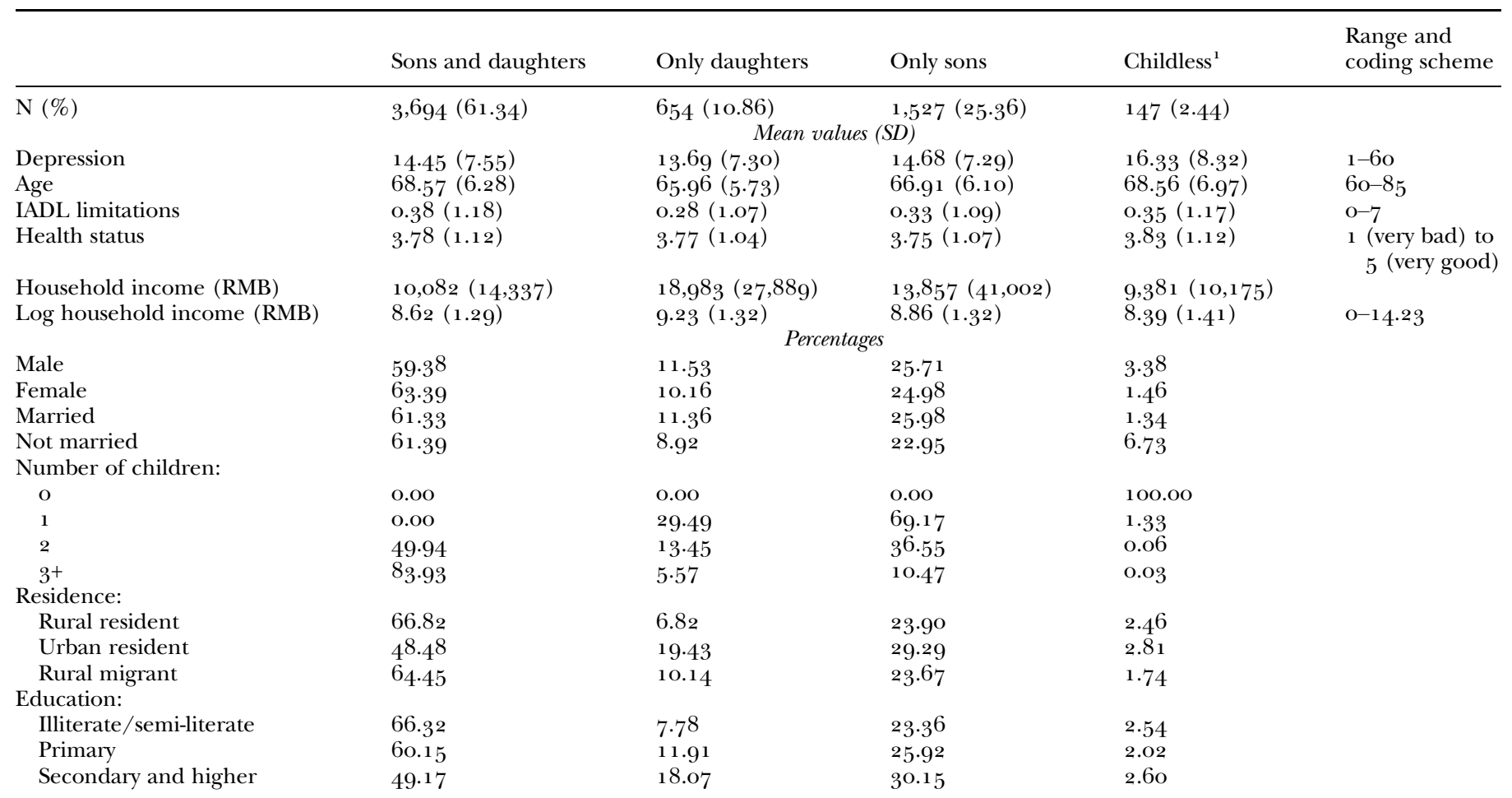




$\begin{array}{lllll}\text { Pension insurance } & 64.26 & 10.27 & 23.55 & 27.68 \\ \text { No pension insurance } & 57.59 & 11.62 & 25 \cdot 33 & 3.92 \\ \text { Han ethnicity } & 61.29 & 10.96 & 25.67 & 2.41 \\ \text { Not Han ethnicity } & 61.99 & 9.44 & 30.29 & 2.91 \\ \text { Retired } & 48.07 & 19.01 & 23.53 & 2.64 \\ \text { Not retired } & 66.27 & 7.83 & 25.48 & 2.37 \\ \text { Religious } & 63.30 & 8.59 & 25.34 & 2.63 \\ \text { Not religious } & 61.08 & 11.17 & 25.17 & 2.42 \\ \text { Family-owned home } & 62.00 & 10.68 & 26.56 & 4.28 \\ \text { Renter } & 57.16 & 12.00 & & \end{array}$

Notes: 1. Includes those who have outlived their children. SD: standard deviation. IADL: instrumental activities of daily living. 
truncating three or more into one category (original variable has mean = 2.92 , standard deviation $=1.49$ ).

Residence. Residence was coded using a combination of the place of living (that indicates whether the respondent lives in an urban or a rural area according to the Census Bureau's definition in 2012), and the respondent's hukou registration (agricultural (rural) or non-agricultural (urban), yielding three categories: rural resident, urban resident and rural migrant.

Other variables. Next to the main variables of interest, we included an array of confounders known from previous research to be associated with depression. Age was used as a continuous indicator and to account for a non-linear relationship with depression we included a quadratic term of age. The gender of parents was binary coded, with men as the reference category. Marital status was measured dichotomously (married versus non-married); there were too few cases of never married and divorced individuals in accordance with previous census and survey data for older adults in China. Education was measured using three categories: a reference category primary education (including illiterate and semi-illiterate), secondary education and tertiary education. Two variables of health status were used: (a) limitations with instrumental activities of daily living, ranging from $o$ to 7 to capture needing help for outdoor activities, eating, kitchen activities, taking public transportation, shopping, cleaning, doing laundry; and (b) self-rated health measured on a scale from o (excellent) to 5 (unhealthy). Ethnicity was measured with a binary indicator, whether respondents belonged to the major ethnic Han group or some other ethnic group. Religious affiliation was measured using a binary indicator that captures whether respondents reported belonging to any denomination (Buddhist, Taoism, Islam, Roman Catholic or Protestant) or reported not being religious.

Due to the complicated nature of capturing all the different types of formal and informal employment in old age, we used a dichotomous measure to indicate if the respondents have officially retired. We used a measure to designate whether individuals currently contribute to an urban or a rural pension insurance scheme, meaning that they need not be currently receiving pension but were covered by some of the types/ schemes of old-age insurance available in China (supplemental pension insurance of firm; commercial pension insurance; Rural Pension Insurance; New Rural Social Pension Insurance; Urban Resident Pension Insurance; Urban-Rural Resident Social Pension Insurance; pension subsidy for the oldest old; or other pension subsidy). Income was measured using the log of adjusted household income per capita due to the skewed 
distribution of the original variable. Lastly, the 25 different provinces were used as fixed-effect dummy variables in the regression models.

We addressed the hypotheses by estimating ordinary least squares (OLS) regression models in order to examine the level of depressive symptoms with data weighted for national representability. In Table 2, the estimates are presented from Models 1 and 2 that test the first and the second hypotheses, and Model 3 that tests the third and fourth hypotheses by including an interaction term for parental status and category of residence. All the models include the confounders known to be associated with depression described in the previous section. To facilitate the interpretation of the coefficients for parental status and the interaction term with category of residence given the multiplicity of categories involved in the analyses, we estimated and plotted marginal effects at representative values for the four groups of parental status: sons and daughters, only sons, only daughters, and sons and daughters.

\section{Results}

\section{Descriptive results}

Table 1 shows descriptive results for the analytical sample. We observe that around two-thirds of the sample have children of both sex, whereas there are far fewer parents of only daughters $(10.86 \%)$ compared to parents of only sons $(25.36 \%)$. This is in line with the established gender imbalance and known son preference reported in the literature (Arnold and Zhaoxiang 1986; Coale 1991; Cleland, Verrall and Vaessen 1983; Johnson 2004; Johnson, Banghan and Liyao 1998). The childless are not numerous (2.44 per cent), a proportion that is consistent with the prevalence of childlessness reported in other general surveys from China (Cheng et al. 2014; Chou and Chi 2004), but still constitutes a lower level of biological childlessness than in other countries (where it is around $5 \%$; see Koropeckyj-Cox and Call 2007). With regards to residence, around half of the whole sample lives in a rural area regardless of their hukou $\left(5^{6.01} \%\right)$, whereas one-third are urban residents with an urban (non-agricultural) hukou (27.78\%). A significant proportion of the older adults are rural migrants $(16.21 \%)$, as they live in the cities but have a rural (agricultural) hukou that puts restrictions on their access to urban welfare provisions.

\section{Multivariate results}

Hypothesis 1 proposes that the childless are more disadvantaged in mental health compared to parents. The findings from Model 1 partially support Hypothesis 1 in that the childless score higher on the CES-D scale compared 
T а в L E 2. Ordinary least squares regressions for depression (Center for Epidemiologic Studies - Depression scale)

\begin{tabular}{|c|c|c|c|c|c|c|}
\hline & \multicolumn{2}{|c|}{ Model 1} & \multicolumn{2}{|c|}{ Model 2} & \multicolumn{2}{|c|}{ Model 3} \\
\hline & $b$ & $\mathrm{SE}$ & $b$ & $\mathrm{SE}$ & $b$ & SE \\
\hline Age & $1.016^{* * *}$ & 0.304 & $0.995^{* *}$ & 0.306 & $1.004^{* * *}$ & 0.304 \\
\hline Age quadratic & $-0.008^{* * * *}$ & 0.002 & $-0.007^{* * *}$ & 0.002 & $-0.008^{* * *}$ & 0.002 \\
\hline Gender & $1.329 * * *$ & 0.197 & $1.33^{* * * *}$ & 0.198 & $1.35^{8 * * *}$ & 0.198 \\
\hline Not married & $1.097 * * *$ & 0.234 & $1.083^{* * *}$ & 0.235 & $1.100 * * *$ & 0.235 \\
\hline \multicolumn{7}{|c|}{ Education (Ref. Illiterate/semi-literate): } \\
\hline Primary & $-0.689^{* *}$ & 0.244 & $-0.689^{* *}$ & 0.244 & $-0.689^{* *}$ & 0.244 \\
\hline Secondary and higher & $-1.078^{* * * *}$ & 0.283 & $-1.074^{* * * *}$ & 0.284 & $-1.033^{* * *}$ & 0.284 \\
\hline Household income log (RMB) & -0.071 & 0.078 & -0.069 & 0.079 & -0.065 & 0.079 \\
\hline IADL limitations & $1.030^{* * *}$ & 0.081 & $1.029 * * *$ & 0.081 & $1.034^{* * *}$ & 0.081 \\
\hline Some pension insurance & $-0.461 *$ & 0.213 & $-0.462 *$ & 0.213 & $-0.441^{*}$ & 0.213 \\
\hline Not Han ethnicity (Other) & $0.45^{6}$ & 0.394 & $0.45^{8}$ & 0.394 & 0.485 & 0.394 \\
\hline Retired & -0.012 & 0.325 & -0.003 & 0.325 & 0.047 & 0.326 \\
\hline \multicolumn{7}{|l|}{ Residence (Ref. Rural): } \\
\hline Urban & $-1.227^{* * *}$ & 0.310 & $-1.217^{* * *}$ & 0.311 & $-0.947^{* *}$ & $0.35^{\circ}$ \\
\hline Rural migrant & $-1.037^{* * *}$ & 0.262 & $-1.035^{* * *}$ & 0.262 & $-0.94^{* *}$ & 0.317 \\
\hline Religious & 0.487 & 0.308 & 0.480 & 0.308 & 0.531 & 0.309 \\
\hline Does not own home & $0.847^{* *}$ & 0.271 & $0.8_{3} 8 * *$ & 0.272 & $0.848^{* *}$ & 0.272 \\
\hline \multicolumn{7}{|c|}{ Parental status (Ref. Sons and daughters): } \\
\hline Only daughters & $0.83^{*}$ & 0.326 & $0.850^{*}$ & 0.345 & $1.494^{* *}$ & 0.507 \\
\hline Only sons & $0.711 * *$ & 0.226 & $0.733^{* *}$ & 0.266 & $0.805^{* *}$ & 0.290 \\
\hline Childless & $1.316^{*}$ & 0.551 & -0.137 & 1.824 & $2.082^{* *}$ & 0.727 \\
\hline \multicolumn{7}{|l|}{ Number of children (Ref. 2): } \\
\hline o & & & 1.626 & 1.911 & & \\
\hline 1 & & & -0.028 & 0.349 & & \\
\hline $3^{+}$ & & & 0.029 & $0.25^{\circ}$ & & \\
\hline
\end{tabular}


Parental status $\times$ Residence:

Only daughters $\times$ Urban

Only daughters $\times$ Rural migrant

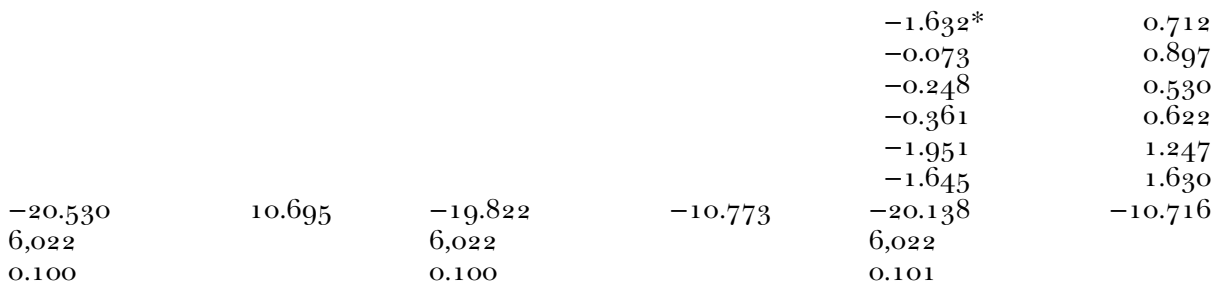

Only sons $\times$ Urban

Only sons $\times$ Rural migrant

Childless $\times$ Urban

Childless $\times$ Rural migrant

Intercept

Observations

0.100

Notes: SE: standard error. Ref.: reference category. IADL: instrumental activities of daily living. Data weighed for national sample representability. Estimates include province fixed effects, coefficients omitted in the table.

Significance levels: $* p<0.05, * * p<0.01, * * * p<0.001$. 
to parents of both sons and daughters $(b=1.316$, standard error $(\mathrm{SE})=$ $\left.0.55^{1}\right)$. In order to facilitate testing of Hypotheses 1 and 2 we present Figure 1, plotting the predictive margins for parental status on depression with 95 per cent confidence intervals (CI). It is noticeable that the childless do not have more depressive symptoms than all parents, but only more than parents who have sons and daughters, thus making the childless similar to the parents of only sons or only daughters. However, an alternative explanation might be that having more children is negatively associated with depressive symptoms regardless of the gender composition of children. Therefore, in Model 2 we tested whether the number of children is predictive of depression, as the specification of parental status does not capture the number of children. Both linear (not shown) and non-linear specifications of the number of children shown in Model 2 revealed that the number of children is not predictive of depressive symptoms per se, prompting that the differences between the childless and the parents are indeed explained by the gender of the children, rather than the number of children. Nonetheless, due to the multicollinearity of the measures in Model 2 (the number of children and children's gender), we excluded the number of children as a measure in Model 3 and used only the children's gender.

With regards to the second hypothesis, we expected that parents who have only daughters will have more depressive symptoms compared to those who had sons (either only sons or both daughters and sons). There is partial support for Hypothesis 2, as from Model 1 it is evident that parents of only daughters have more depressive symptoms than parents who have both sons and daughters $(b=0.836, \mathrm{SE}=0.326)$ and the association is even stronger when the model includes the number for children (Model 2). In Figure 1 we observe that parents of only daughters do not score higher on the depression measure than parents of only sons $\left(b=0.15^{1}, \mathrm{SE}=0.299\right)$. Even in Model 2 where the number of children is included, parents of only daughters are not more depressed than parents of only sons.

To investigate the role of the rural-urban divide and the impact modernisation might have on the mental health of the older childless, we proceeded to check whether differences exist between groups living in different circumstances with regards to their residence and privileges that the household registration system confers. We expected that the difference in the prevalence of depressive symptoms would be greater between the childless and parents amongst rural and migrant populations than amongst those with urban residence (Hypothesis 3). In Model 3, those with urban residence have fewer depressive symptoms compared to parents of only daughters with rural residence $(b=-1.632, \mathrm{SE}=0.712)$, thus granting some support for Hypothesis 3. To observe differences in depression levels between groups with different residence status, we 
17

16

15

14

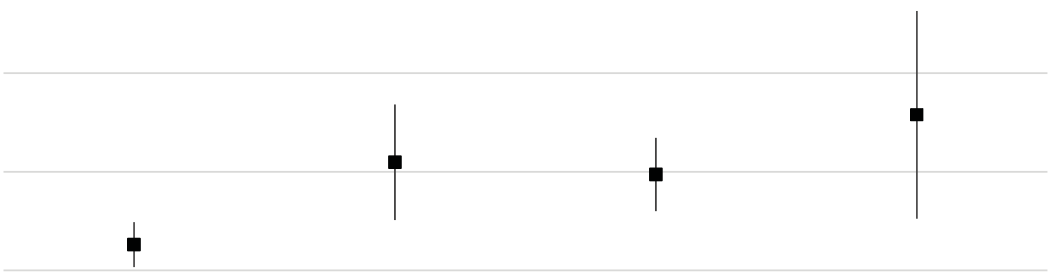

13

12

Sons and daughters $\quad$ Only daughters $\quad$ Only sons $\quad$ Childless

Figure 1. Predictive margins for parental status on parental depression with 95 per cent confidence intervals.

Note: The depression score is measured on the Center for Epidemiologic Studies - Depression scale, with a higher score indicating more depressive symptoms.

plotted the marginal effects of the interaction between parental status and residence with 95 per cent CI (Figure 2).

Notably, the rural childless do not score higher on the CES-D scale than the urban childless or the childless migrants, contrary to our expectations in Hypothesis 3. However, Model 3 lends support for Hypothesis 4 as older adults with only daughters are more disadvantaged in rural areas compared to the urban parents of only daughters, but fare the same as rural migrants with only daughters. Note that this result is dependent on the number of children as it does not hold when single-child families are excluded. In that case the difference between urban residents with only daughters and rural residents with only daughters diminishes. In addition, we notice striking similarities between groups who have only daughters and the childless. Both the childless rural and the rural parents of only daughters have more depressive symptoms compared to the rural parents who have sons and daughters; but the groups do not differ between each other. On the other side, there are no differences between the urban parents and the urban childless, suggesting that the disadvantage of the childless and the parents of only daughters is pertinent to the rural context where children function as an old-age security system for older parents. Sensitivity analyses, shown in supplementary online material, reveal that the results are not sensitive to gender, age and marital status when interaction terms between parental status and each of the variables was included in models. 


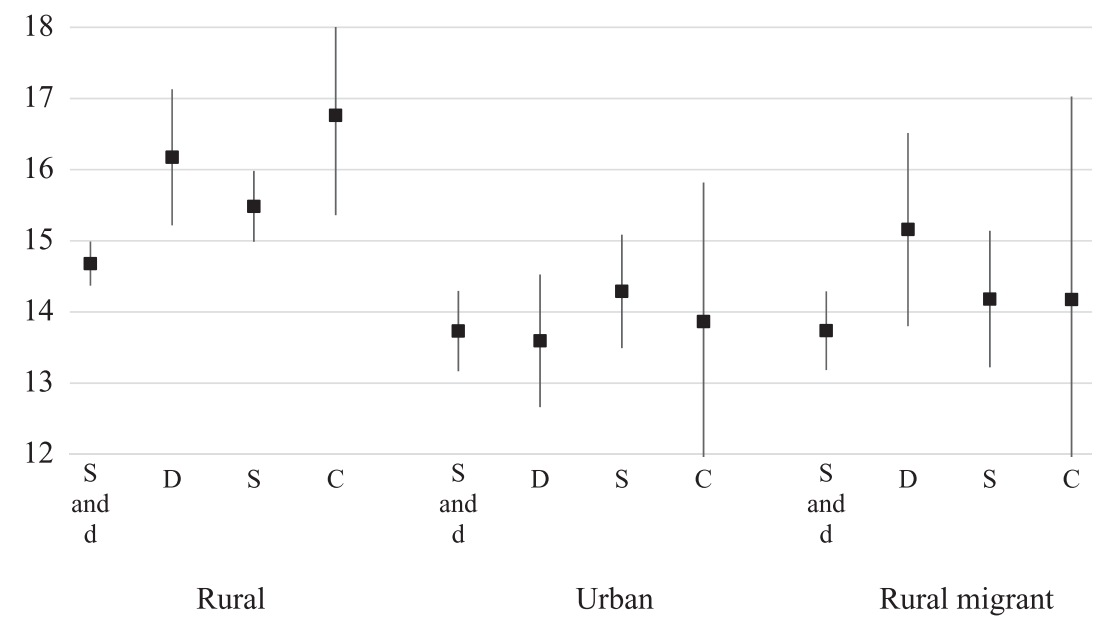

Figure 2. Predictive margins for the interaction effect of parental status and residence on parental depression with 95 per cent confidence intervals.

Notes: The depression score is measured on the Center for Epidemiologic Studies - Depression scale, with a higher score indicating more depressive symptoms. S and d: sons and daughters. D: only daughters. S: only sons. C: childless.

\section{Discussion}

We set out to establish how the mental wellbeing of the childless in a pronatalist society such as the Chinese compares not only to parents, but to parents of children with different gender composition. China is uniquely different with a remarkably stronger preference for sons compared to European societies and the United States of America (USA) where there is a strong preference for a mixed-sex composition (Das Gupta et al. 2003; Hank and Kohler 2000). Previous studies touching upon the relationship between childlessness and depression have focused on the number of children older adults have (in Europe, Buber and Engelhardt 2008), or just compared the childless to all parents in the USA (Bures, Koropeckyj-Cox and Loree 2009) and in China (Chou and Chi 2004; Guo 2014). Previous research has shown that the childless are not more depressed than parents in the USA (Evenson and Simon 2005; Koropeckyj-Cox, Pienta and Brown 2007) and across Europe (Hank and Wagner 2013). Other studies report worse mental health for older childless individuals in the USA (Koropeckyj-Cox 1998), while Huijts, Kraaykamp and Subramanian (2013) found that depression levels of childless people in 24 European countries are smaller in countries with tolerant norms towards childlessness and high levels of social support. A study that has looked into childless men, in particular, found a relationship between widowhood and low wellbeing underlying the relationship between childlessness and wellbeing (Dykstra and Keizer 2009). 
Our findings showed that despite a son preference in China, when it comes to mental health just having a son is not what matters as the best faring groups with lower depression levels are parents that have both sons and daughters. Although childlessness was very low, the increasing rates of divorce, the postponement of parenthood and the decrease in birthrates might lead to a larger number of individuals remaining involuntarily childless in the future. The neglect of childless people in countries such as China has been largely due to the perception of the childless as a disadvantaged set of isolated and presumably marginalised people. The childless have therefore not appeared to be overly problematic as welfare provision or kin networks have been assumed to be adequate. However, especially in rural areas, it seems that there is not yet an adequate substitute for adult children to care for the needs of the older population (Chen and Silverstein 2000; Cong and Silverstein 2008; Guo 2014; Guo, Aranda and Silverstein 2009; Guo, Chi and Silverstein 2012, 2016). The rising prevalence of childlessness should prompt further research into this group.

Furthermore, the fact that sonless parents are similar to the childless in rural areas (and whose proportion is much higher in the population than those who are childless) indicates that children's gender composition should be far more central in such research. The groups should be treated as a sub-population of general demographic and sociological importance, rather than an isolated minority that has been marginalised as an abnormal phenomenon of concern chiefly to special health and welfare policies. With regards to the group of older adults that have only sons, due to skewed sex ratios in the future there is a danger that the proportion of this group in the population will rise. A danger related to this is that due to a marriage squeeze the sons might not be able to find wives and older parents may end up living with bachelor sons. This in turn might be related negatively with the mental health of such parents because of the strong cultural expectation that daughters-in-law should take care of older adults.

Despite the literature dealing with dependency ratios and measures of coresidence, studies have failed to capture the diverse circumstances of older adults such as the difference in opportunities in urban and rural areas in China. In the literature on childlessness in the West, they are thought to have adapted to their status and some of them live their lives without assistance and in most cases siblings and other kin exist who may be able to help when necessary. Yet not much is known about the role of other relatives in the support networks of the childless old in China. However, our study shows that unlike in the West, where a large proportion of the childless old are well off and a minority of potential welfare concern, in China older adults without children are vulnerable to suffer from poor mental health. 
The real need is to identify those parts of the older population that face a serious lack of assistance and those who do not, and this study goes some way to addressing this need. The childless may be divided like all other people into two blocks: those with sufficient access to resources such as commercial-sector pensions and health care, and those dependent on assistance, either from kin or the state (Kraeger 2004). Theoretically, and as demonstrated empirically, older adults' lives in China need to be investigated in a historical context. We expanded on previous work looking at the heterogeneity in childless adults and how it might be related to wellbeing that have focused mostly on marital history differences (Chou and Chi 2004; Dykstra and Keizer 2009) by turning to residence and migration status as key indicators important for both mental health and parental status in a society as vast and as diverse as China. Our findings identified a few particularly vulnerable groups such as rural residents without any sons and the childless rural residents. The results showed that rural residents with only daughters have more depressive symptoms than rural residents with daughters and sons when all families are taken in consideration.

Notably, as a part of the sensitivity analyses shown in the supplementary online material, we did not find gender differences between parents when it comes to the relationship between parental status and depression; although in societies with a strong pronatalist culture, childlessness might threaten older woman's health more than men's (Daniluk 2003). Our findings did not support this assumption, as parental status was equally predictive of men's and women's depression levels. The finding is not consistent with US studies that found that divorced childless men had the highest odds of depression (Zhang and Heyward 2001). One limitation of this study that we have to accept is that we cannot look at those who never had children separately from those who have outlived their offspring due to the very low proportion of the latter. Parents who have outlived their children might do better or worse compared to never-parents, depending on how they have adjusted to their childless status. The second limitation is that we do not include the transfers made between the parents and children, but draw on the mere presence of children in parents' lives, and we disregard the possible quality of the relationship between the adult child and parent.

In sum, the available evidence suggests that modernisation plays a role in the relationship between mental health and parenthood in late life, as results differ significantly between urban and rural residents, with the rural migrants not faring as badly as expected when it comes to depression. Showing a sensitivity to the social context in China, we investigated parental status' association with mental health to see whether negative health consequences were greater for those with children of the 'unwanted gender'. Results confirmed that parents with only daughters were disadvantaged in 
a manner similar to the childless in rural areas, but not in urban areas. Despite the preference for sons, it is evident that the groups who are worse off are indeed those who have only daughters. Yet having only sons does not necessarily lead to greater wellbeing. In a context where the relationship between parents and children is undergoing changes, values and expectations do not go hand in hand with that change. Future research should consider how the effects of childlessness may change over time as well as across social groups and cohorts (Dykstra 2016).

\section{Acknowledgement}

The research leading to these results has received funding from the ESRC-funded research project 'Life Course and Family Dynamics in a Comparative Perspective' (ES/Lo15927/1). No ethical approval was requested for the study.

\section{Supplementary Material}

To view supplementary material for this article, please visit https://doi.org/ $10.1017 /$ So $144686 \mathrm{X}_{17} 700054 \mathrm{X}$

\section{References}

Arnold, F. and Kuo, E. C.Y. 1984. The value of daughters and sons: a comparative study of the gender preferences of parents. Journal of Comparative Family Studies, 15, 2, 299-318.

Arnold, F. and Zhaoxiang, L. 1986. Sex preference, fertility, and family planning in China. Population and Development Review, 1 2, 2, 221-46.

Bian, F., Logan, J. R. and Bian, Y. 1998. Intergenerational relations in urban China: proximity, contact, and help to parents. Demography, 35, 1, $115^{-24}$.

Buber, I. and Engelhardt, H. 2008. Children's impact on the mental health of their older mothers and fathers: findings from the Survey of Health, Ageing and Retirement in Europe. European Journal of Ageing, 5, 1, 31-45.

Bures, R. M., Koropeckyj-Cox, T. and Loree, M. 2009. Childlessness, parenthood, and depressive symptoms among middle-aged and older adults. Journal of Family Issues, 30, 5, 670-87.

Central Intelligence Agency 2015. China. In The World Factbook. Available online at https://www.cia.gov/library/publications/the-world-factbook/geos/ch.html [Accessed 3 October 2016].

CESD-R Explanation 2008. The Centre for Epidemiological Studies Depression Scale Revised. Available online at http://cesd-r.com/cesdr/ [Accessed 3 October 2016].

Chan, K. W. and Zhang, L. 1999. The hukou system and rural-urban migration in China: processes and changes. The China Quarterly, 16o, 818-55.

Chen, R., Copeland, J. R. M. and Wei, L. 1999. A meta-analysis of epidemiological studies in depression of older people in the People's Republic of China. International Journal of Geriatric Psychiatry, 14, 10, 821-30. 
Chen, J., Xie, Z. and Liu, H. 2007. Son preference, use of maternal health care, and infant mortality in rural China, 1989-2000. Population Studies, 61, 2, 161-83.

Chen, X. and Silverstein, M. 2000. Intergenerational social support and the psychological well-being of older parents in China. Research on Aging, 22, 1, 43-65.

Cheng, S. T., Chan, T. W. S., Li, G. H. and Leung, E. M. 2014. Childlessness and subjective well-being in Chinese widowed persons. Journals of Gerontology: Psychological Sciences and Social Sciences, 69B, 1, 48-52.

Chou, K. L. and Chi, I. 2004. Childlessness and psychological well-being in Chinese older adults. International Journal of Geriatric Psychiatry, 19, 5, 449-7.

Chu, C. C. 1991. Primogeniture. Journal of Political Economy, 99, 1, 78-99.

Chu, C. Y. and Yu, R. R. 20o9. Co-residence and family size. In Chu, C. Y. and Yu, R. R. (eds), Understanding Chinese Families: A Comparative Study of Taiwan and Southeast China. Oxford University Press, Oxford, $130-32$.

Chu, C. Y., Xie, Y. and Yu, R. R. 2011 . Coresidence with elderly parents: a comparative study of southeast China and Taiwan. Journal of Marriage and Family, 73, 1, 120-35.

Cleland, J., Verrall, J. and Vaessen, M. 1983. Preferences for the Sex of Children and Their Influence on Reproductive Behaviour. World Fertility Surveys Comparative Studies 27, International Statistical Institute, Voorburg, The Netherlands.

Coale, A. J. 1991. Excess female mortality and the balance of the sexes in the population: an estimate of the number of 'missing females'. The Population and Development Review, 17, 3, 517-23.

Cong, Z. and Silverstein, M. 2008. Intergenerational support and depression among elders in rural China: do daughters-in-law matter? Journal of Marriage and Family, 7o, 3, 599-612.

Cong, Z. and Silverstein, M. 2011 . Parents' depressive symptoms and support from sons and daughters in rural China. International Journal of Social Welfare, 2o, 1, 4-1 7 .

Cong, Z. and Silverstein, M. 2011 . Intergenerational exchange between parents and migrant and nonmigrant sons in rural China. Journal of Marriage and Family, 73, 1, 93-104.

Cong, Z. and Silverstein, M. 2012. A vignette study on gendered filial expectations of elders in rural China. Journal of Marriage and Family, 74, 3, $5^{10-25}$.

Cooney, R.S. and Di, J. 1999. Primary family caregivers of impaired elderly in Shanghai-China: kin relationship and caregiver burden. Research on Aging, 2 1, 6, 739-61.

Daniluk, J. C. 2003. Women's Sexuality Across the Life Span: Challenging Myths, Creating Meanings. Guilford Press, New York.

Das Gupta, M., Zhenghua, J., Bohua, L., Zhenming, X., Chung, W. and Hwa-Ok, B. 2003. Why is son preference so persistent in East and South Asia? A cross-country study of China, India and the Republic of Korea. Journal of Development Studies, 4o, 2, 153-87.

Djundeva, M., Mills, M., Wittek, R. and Steverink, N. 2015. Receiving instrumental support in late parent-child relationships and parental depression. Journals of Gerontology: Psychological Sciences and Social Sciences, 7oB, 6, 981-94.

Dykstra, P. A. 2016. Childless older adults. In Whitbourne, S. K. (ed.), Encyclopedia of Adulthood and Aging. Wiley-Blackwell, Hoboken, New Jersey, 1-4.

Dykstra, P.A. and Hagestad, G. O. 2007. Roads less taken: developing a nuanced view of older adults without children. Journal of Family Issues, 28, $10,1275^{-3} 10$.

Dykstra, P. A. and Keizer, R. 2009. The wellbeing of childless men and fathers in midlife. Ageing $\mathcal{E}$ Society, 29, 8, $1227-42$.

Evenson, R. J. and Simon, R. W. 2005. Clarifying the relationship between parenthood and depression. Journal of Health and Social Behavior, 46, 4, 341-58. 
Feng, X., Guo, L. and Liu, K. 2016. Reliability and construct validity of cut version of Center for Epidemiological Studies Depression Scale (CES-D) in community elderly population. China Journal of Modern Medicine, 28, 10, 131-34.

Feng, Z., Liu, C., Guan, X. and Mor, V. 2012. China's rapidly aging population creates policy challenges in shaping a viable long-term care system. Health Affairs, 31, 1 2, 2764-73.

Friedman, D., Hechter, M. and Kanazawa, S. 1994. A theory of the value of children. Demography, 31, 3, 375-401.

Gao, S., Jin, Y., Unverzagt, F. W., Liang, C., Hall, K. S., Ma, F., Murrell, J. R., Cheng, Y., Matesan, J., Li, P., Hendrie, H. C. and Bian, J. 2009. Correlates of depressive symptoms in rural elderly Chinese. International Journal of Geriatric Psychiatry, 24, 12, 1358-66.

Giles, J. and Mu, R. 2007. Elderly parent health and the migration decisions of adult children: evidence from rural China. Demography, 44, 2, 265-88.

Guo, M. 2014. Parental status and late-life well-being in rural China: the benefits of having multiple children. Aging and Mental Health, 18, 1, 19-29.

Guo, M., Aranda, M. P. and Silverstein, M. 2009. The impact of out-migration on the inter-generational support and psychological wellbeing of older adults in rural China. Ageing E Society, 29, 7, $1085^{-104}$.

Guo, M., Chi, I. and Silverstein, M. 2012. The structure of intergenerational relations in rural China: a latent class analysis. Journal of Marriage and Family, 74, 5, $1114^{-28}$.

Guo, M., Chi, I. and Silverstein, M. 2016. Trajectories and determinants of elder care in rural China during an 8-year period: why having sons makes a difference. Research on Aging, 38, 5, 531-53.

Hank, K. and Kohler, H. P. 200o. Gender preferences for children in Europe: empirical results from 17 FFS countries. Demographic Research, 2, 1, 1-21.

Hank, K. and Wagner, M. 2013. Parenthood, marital status, and well-being in later life: evidence from SHARE. Social Indicators Research, 1 1, 42, 639-53.

Hesketh, T. and Wei, X. Z. 1997. Health in China. From Mao to market reform. BMJ: British Medical Journal, 314, 7093, 1543.

Huijts, T., Kraaykamp, G. and Subramanian, S. V. 2013. Childlessness and psychological well-being in context: a multilevel study on 24 European countries. European Sociological Review, 29, 1, 32-47.

Institute of Social Science Survey. 201 2. China Family Panel Studies Follow-up Survey, Harvard Dataverse, V1. http://dx.doi.org/10.7910/DVN/WLCDYJ

Johnson, K. A. 2004. Wanting a Daughter, Needing a Son: Abandonment, Adoption, and Orphanage Care in China. Yeong and Yeong, St. Paul.

Johnson, K., Banghan, H. and Liyao, W. 1998. Infant abandonment and adoption in China. Population and Development Review, 24, 3, 469-510.

Koropeckyj-Cox, T. 1998. Loneliness and depression in middle and old age: are the childless more vulnerable? Journals of Gerontology: Psychological Sciences and Social Sciences, 53B, 6, S303-12.

Koropeckyj-Cox, T. and Call, V. R. 2007. Characteristics of older childless persons and parents: cross-national comparisons. Journal of Family Issues, 28, 10, 1362-414.

Koropeckyj-Cox, T., Pienta, A. M. and Brown, T. H. 2007. Women of the 1950 os and the 'normative' life course: the implications of childlessness, fertility timing, and marital status for psychological well-being in late midlife. International Journal of Aging and Human Development, 64, 4, 299-330.

Kraeger, P. 2004. Where are the children? In Kraeger, P. and Schroeder-Butterfill, E. (eds), Ageing Without Children: Asian and European Perspectives. Berghahn, Oxford, 1-45.

Lai, D., Tong, H., Zeng, Q. and Xu, W. 2010. The factor structure of a Chinese Geriatric Depression Scale-SF: use with alone elderly Chinese in Shanghai, China. International Journal of Geriatric Psychiatry, 25, 5, 503-10. 


\section{Maja Djundeva et al.}

Lee, Y.J. and Xiao, Z. 1998. Children's support for elderly parents in urban and rural China: results from a national survey. Journal of Cross-cultural Gerontology, 13, 1, 39-62.

Lim, L. L., Chang, W., Yu, X., Chiu, H., Chong, M.Y. and Kua, E. H. 2011. Depression in Chinese elderly populations. Asia-Pacific Psychiatry, 32, 46-53.

Liu, H.J., Meng, C. and Tang, Z. 2004. Survey on depression of Beijing residents over 55 year-old. Chinese Mental Health Journal, 18, 794-95.

Liu, Y., Hsiao, W. C. and Eggleston, K. 1999. Equity in health and health care: the Chinese experience. Social Science and Medicine, 49, 10, 1349-5.

Lui, F. T. 2010. Demographic transition, childless families, and economic growth. In Ito, T. and Rose, A. K. (eds), The Economic Consequences of Demographic Change in East Asia, NBER-EASE. Volume 19, University of Chicago Press, Chicago, 351-73.

Luo, G. 2012. Social policy, family support, and rural elder care. In Chen, S. and Powell, J. L. (eds), Aging in China. Springer, New York, 83-98.

Pan, A., Sun, Q., Okereke, O. I., Rexrode, K. M. and Hu, F. B. 2011 . Depression and risk of stroke morbidity and mortality: a meta-analysis and systematic review. JAMA, 306, $11,1241-9$.

Pei, X. and Tang, Y. 2012. Rural old age support in transitional China: efforts between family and state. In Chen, S. and Powell, J. L. (eds), Aging in China. Springer, New York, 61-81.

Poston, D. L. 2002. Son preference and fertility in China. Journal of Biosocial Science, 343, 333-47.

Poston, D. L. and Glover, K. S. 2005. Too many males: marriage market implications of gender imbalances in China. Genus, 61, 2, 119-40.

Potts, M. 2006. China's one child policy. BMJ, 333, 7564, $3^{61-2 .}$

Radloff, L. S. 1977. The CES-D scale: a self-report depression scale for research in the general population. Applied Psychological Measurement, 1, 3, 385-401.

Shen, C. and Williamson, J. B. 2010. China's new rural pension scheme: can it be improved? International Journal of Sociology and Social Policy, 3o, 5/6, 239-5o.

Silverstein, M., Chen, X. and Heller, K. 1996. Too much of a good thing? Intergenerational social support and the psychological well-being of older parents. Journal of Marriage and the Family, 58, 4, 970-82.

Song, S. 2009. Does famine have a long-term effect on cohort mortality? Evidence from the 1959-1961 Great Leap Forward famine in China. Journal of Biosocial Science, 41, 4, 469-91.

Stek, M. L., Gussekloo, J., Beekman, A. T. F., Van Tilburg, W. and Westendorp, R. G. J. 2004. Prevalence, correlates and recognition of depression in the oldest old: the Leiden 85-plus study. Journal of Affective Disorders, 78, 3, 193-200.

Sun, R. 2002. Old age support in contemporary urban China from both parents' and children's perspectives. Research on Aging, 24, 3, 337-59.

Tiikkainen, P. and Heikkinen, R. L. 2005. Associations between loneliness, depressive symptoms and perceived togetherness in older people. Aging and Mental Health, 9, 6, 526-34.

United Nations 2007. World Population Ageing 2007. Population Division, United Nations Department of Economic and Social Affairs, New York.

United Nations 2008. World Population Prospects, the 2008 Revision. Population Division, United Nations Department of Economic and Social Affairs, New York.

Wang, L. N., Tang, D., Xu, J.J. and Shen, J. L. 2006. The sex difference of effect of social support on depressive symptoms of the elderly in China. Chinese Journal of Clinical Psychology, 14, 1, 70-84.

Wen, Z., Bi-Rong, D., Chang-Quan, H., Zhen-Chan, L., Yuan, Z., Hong-Mei, W., YanLing, Z., Hui, W. and Ping, H. 2010. Depression and hypertension among Chinese nonagenarians and centenarians. International Journal of Geriatric Psychiatry, 25, 6, 554-61. 
Whyte, M. 2004. Filial obligations in Chinese families: paradoxes of modernization. In Ikels, C. (ed.), Filial Piety: Practice and Discourse in Contemporary East Asia. Stanford University Press, Stanford, California, 106-27.

Whyte, M. 2005. Continuity and change in Chinese family life. The China Journal, 53 , 9-33.

Whyte, M. K. 2010. One Country, Two Societies: Rural-Urban Inequality in Contemporary China. Volume 16, Harvard University Press, Cambridge, Massachusetts.

Wu, X. and Treiman, D. J. 2004. The household registration system and social stratification in China: 1955-1996. Demography, 41, 2, 363-84.

Wulsin, L. R., Vaillant, G. E. and Wells, V. E. 1999. A systematic review of the mortality of depression. Psychosomatic Medicine, 61, 1, 6-1 7 .

Xie, Y. and Zhu, H. 2009. Do sons or daughters give more money to parents in urban China? Journal of Marriage and Family, 71, 1, 174-86.

Yi, Z., Vaupel, J. W. and Yashin, A. I. 1985 . Marriage and fertility in China: a graphical analysis. Population and Development Review, 11, 4, 721-36.

Yip, W. and Hsiao, W. 2009. China's health care reform: a tentative assessment. China Economic Review, 20, 4, 613-9.

Zeng, Y., Brasher, M. S., Gu, D. and Vaupel, J. W. 2016. Older parents benefit more in health outcome from daughters' than sons' emotional care in China. Journal of Aging and Health, 28, 8, 1426-47.

Zhan, H.J. 2004. Willingness and expectations: intergenerational differences in attitudes toward filial responsibility in China. Marriage and Family Review, 36, 1/2, $175^{-200 .}$

Zhan, H. J., Luo, B. and Chen, Z. 201 2. Institutional elder care in China. In Chen, S. and Powell, J. L. (eds), Aging in China. Springer, New York, 22 1-35.

Zhang, Z. and Hayward, M. D. 2001. Childlessness and the psychological well-being of older persons. The Journals of Gerontology Series B: Psychological Sciences and Social Sciences, 56, 5, 311-20.

Zhang, B., and Li, J. 2011 . Reliability and validity of a short version of Center for Epidemiologic Studies Depression Scale in national adult population. Chinese Mental Health Journal, 25, 7, 506-1 1.

Zhang, J., Wu, Z. Y., Fang, G., Li, J., Han, B. X. and Chen, Z. Y. 2010 . Development of the Chinese age norms of CES-D in urban area. Chinese Mental Health Journal, 24, 2, 139-43.

Zhang, W. and Liu, G. 2007. Childlessness, psychological well-being, and life satisfaction among the elderly in China. Journal of Cross-cultural Gerontology, 22, 2, $185^{-203}$.

Zhang, W. and Wang, Y. 2010. Meal and residence rotation of elderly parents in contemporary rural Northern China. Journal of Cross-cultural Gerontology, 25, 3, 2 1 7-37.

Accepted I9 April 2017

Address for correspondence:

Maja Djundeva,

Department of Public Administration and Sociology,

Erasmus University Rotterdam,

3062 PA Rotterdam, The Netherlands

E-mail: djundeva@fsw.eur.nl 